\title{
Cooperation and Coordination in Cognitive Networks with Packet Retransmission
}

\author{
Marco Levorato*, Osvaldo Simeone $\ddagger$, Urbashi Mitra ${ }^{\dagger}$, Michele Zorzi* \\ * Dept. of Information Engineering, University of Padova, via Gradenigo 6/B, 35131 Padova, Italy. \\ $\dagger$ Dept. of Electrical Engineering, New Jersey Institute of Technology, Newark, USA. \\ $\ddagger$ Dept. of Electrical Engineering, University of Southern California, Los Angeles, USA. \\ e-mail: \{levorato,zorzi\}@dei.unipd.it, osvaldo.simeone@njit.edu, ubli@usc.edu.
}

\begin{abstract}
The cognitive framework, which fosters an efficient use of the channel resource by granting access to smart and adaptive terminals, has been recently integrated with the cooperative paradigm. Accordingly, following a spectrum-leasing approach, licensed (primary) users may let unlicensed (secondary) users access the owned bandwidth in exchange for an increased performance via cooperation. Nevertheless, assuming that secondary transmission is limited to idle primary slots, as the traffic from the primary sources increases, implementation of spectrum leasing via cooperation becomes more challenging. In fact, the opportunities for secondary transmission reduce and the secondary users may find spectrum leasing not advantageous. In this paper, a solution is proposed that exploits fading temporal correlation and retransmission-based error control in order to increase secondary throughput even in high primary traffic conditions. Focusing on a simple network with a primary and a secondary source transmitting to a common destination, it is shown that the primary source may actively leave part of its channel resource to the secondary source without significant performance loss while greatly improving secondary throughput.
\end{abstract}

\section{INTRODUCTION}

Following Mitola's original definition [1], cognitive radio integrates computational intelligence and machine learning in wireless devices to improve their adaptability to the surrounding environment. Since then, this definition has been extended to include theoretical frameworks aimed at the solution of problems requiring device adaptability and dynamic resource allocation. A scenario that has been the subject of a flourishing interest targets the exploitation of smart unlicensed terminals that access opportunistically available channel resource in order to increase bandwidth usage efficiency [2]-[4]. Some prior work studies concurrent transmission by secondary and primary users in a highly dynamic environment [5], [6].

Recently, the cognitive framework applied to this broad class of problems has been integrated with the cooperative communications paradigm [7]. Cooperation [8] is a widely investigated technique to provide channel diversity in slowlyvarying channels. The essence of cooperation is to let nodes that are not directly involved in a communication between a given source-destination pair transmit data frames associated with the information content sent by the original source. In the context of cognitive radio networks, this idea can be used as a way to implement spectrum leasing [7]. Specifically, the licensed (primary) users may lease part of their available bandwidth to unlicensed (secondary) users in exchange for cooperation.

In the spectrum-leasing approach of [9], secondary sources act as cooperators for primary users' communications, and pay

The activity of Dr. Simeone has been supported by the U.S. National Science Foundation under grant CCF-0914899.

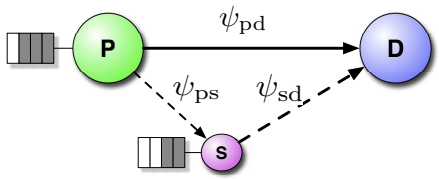

Fig. 1. Three-node topology with a primary and a secondary source, denoted with $\mathbf{P}$ and $\mathbf{S}$, respectively, and the common destination $\mathbf{D}$.

for the opportunity to access the channel by sacrificing part of the channel resource left unused by primary users to forward (relay) primary packets. On the one hand, cooperation by secondary users is required to provide a performance gain to primary communications that is sufficient to justify the lease. On the other hand, a secondary user may not be interested in investing its battery or processing resources if the spectrum leased is insufficient. If we assume, as most prior work on cognitive radios, that secondary users access the channel in the so called white spaces, i.e., frequency-time resource left unused by primary users, then, as primary traffic increases, transmission opportunities for secondary users become rarer. In this case, the effectiveness of cooperation taking place opportunistically in the white spaces is frustrated by the poor chance a secondary user has to be a relay. In [9], this problem is solved by statically assigning a fraction of the time resource to secondary user operations. In this case, spectrum leasing is profitable for primary users only if the benefit of secondary users' cooperation overcomes the loss due to (static) bandwidth splitting.

In this paper, we address the problem outlined above and propose a solution based on the exploitation of temporal fading correlation and retransmission based error control. We show that the primary users can release part of its transmission resources to the secondary users in an opportunistic fashion, so that the primary performance gain is the same, if not greater, than that achieved with opportunistic secondary users cooperation, while the performance achieved by the latter class of users is hugely improved. We refer to this approach as coordinated. The proposed scheme is similar to [10] but, unlike the latter, herein the decision to relinquish transmission opportunities to the secondary nodes is performed by using only locally available information at the primary user by leveraging channel memory, i.e., no explicit message exchange is needed between primary and secondary nodes.

\section{System Description}

We consider the three-node configuration depicted in Fig. 1, where a primary and a secondary source, referred to as nodes $\mathbf{P}$ and $\mathbf{S}$, respectively, send packets to a common destination D. Time is divided into slots of fixed duration, and each slot 
has a data and a feedback phase. In the data phase, one of the sources transmits a data frame to $\mathbf{D}$. The destination then sends, in the feedback phase, a short control packet to either acknowledge (ACK packet) or not acknowledge (NACK) the previously received packet. The channel fading processes are time correlated. In particular, we model the channel coefficients of adjacent slots as correlated Rayleigh variables with correlation equal to $\rho$. We adopt a threshold model for packet decoding, i.e., a packet is successfully decoded if the signalto-noise-ratio (SNR) in the slot is above a rate-dependent threshold $\tau(R)$, where $R$ denotes the transmission rate.

We model the primary source $\mathbf{P}$ as a "dumb" node, employing a static strategy to deliver the packets in its queue. When $\mathbf{P}$ has a non-empty queue, it accesses the slot and transmits a packet with fixed transmission rate equal to $R_{p}$ [bits/s/Hz]. For the sake of simplicity, we assume that the transmission of a single packet at rate $R_{p}$ fits the duration of the data phase. In order to reduce the probability of packet delivery failure, $\mathbf{P}$ adopts a retransmission-based error control scheme. By this scheme, upon the reception of a NACK by the destination, $\mathbf{P}$ schedules a further transmission of the packet sent in the current slot after a fixed delay equal to $M$ slots. Delayed retransmission is a widely used strategy in correlated channels. In fact, by spreading retransmissions over time, the correlation of the channel conditions associated with different transmissions of the same packet is reduced, and the probability of successful packet delivery increases. Conversely, if the retransmissions are concentrated in a short time-span, they are likely to be affected by fading-dip bursts.

In the following we describe the different protocols we consider for the integration of the secondary source $\mathbf{S}$ in the system.

\section{A. Non-cooperative Scenario}

In the non-cooperative scenario, the secondary source is an opportunistic terminal that exploits slots left unused by the primary source to transmit packets to $\mathbf{D}$. Thus, when $\mathbf{S}$ is willing to send a packet, it senses the channel at the beginning of the slot and transmits a packet whenever it perceives the channel as idle. Unlicensed users are assumed to be "smart" terminals, that try to best exploit the available channel resource. Therefore, we implement, at the secondary source a rate adaptation scheme, that bases the current transmission rate on previous measurements of the channel conditions. In particular, $\mathbf{S}$ measures the SNR every time the destination sends an ACK/NACK and computes the associated transmission rate. In the next available slot, $\mathbf{S}$ transmits at rate $R_{s}$ [bit/s/hz], equal to the greatest rate supported by the previously measured SNR. We observe that secondary users are not likely to have strict quality of service requirements in terms of delay or packet delivery probability. Thus, we do not implement the retransmission mechanism at the secondary source.

It is worth remarking that, as channel occupation due to primary source access increases, transmission opportunities for the secondary source get rarer. Note that the primary source accesses slots to either transmit fresh packets or retransmit those packets that incurred failure. Thus, the total channel occupation is a function of both the arrival rate and transmission failure.

\section{B. Cooperative Scenario}

Consider now a scenario where the secondary user can access the channel in idle slots, but only under the condition that it gives priority to cooperative data forwarded on behalf of the primary users. Notice that this model does not explicitly capture secondary quality-of-service requirements (unlike [9] [10]). In our dynamic model, we assume that $\mathbf{S}$ has a cooperation-buffer of size one, where the last packet sent by the source that received a NACK and successfully decoded by $\mathbf{S}$ is stored. When $\mathbf{S}$ senses an idle channel, it checks its cooperation-buffer. If the latter is empty, it proceeds as in the non-cooperative case, and only transmits data from its own queue at the estimated maximum rate $R_{p}$ based on the previously received signal from $\mathbf{D}$. If $\mathbf{S}$ has a packet in its cooperation-buffer and $R_{s}>R_{p}$, the packet from $\mathbf{P}$ is relayed at rate $R_{s}$, as per the spectrum leasing rule discussed above, and the remaining part of the slot is used by $\mathbf{S}$ to send a packet (at variable rate) from its own queue. If the cooperative transmission succeeds, the destination sends the ACK to both $\mathbf{P}$ and $\mathbf{S}$, and the former cancels the previously scheduled retransmission. Nevertheless, the slot reserved for packet retransmission may be used by $\mathbf{P}$ to transmit a packet from its own queue. Note that cooperative transmission by $\mathbf{S}$ may reduce the delay incurred by primary packets due to channel correlation and the consequent delayed retransmission policy.

\section{Coordinated Scenario}

As traffic due to primary source transmissions increases, the probability that $\mathbf{S}$ finds an empty slot gets smaller. In the cooperative scenario described above, this also means that the primary performance gain due to secondary cooperation diminishes. We propose a simple protocol that effectively deals with this issue by exploiting channel memory and the retransmission policy. We call it coordinated, because it requires specific actions by the primary source, that can be seen as a form of coordination with secondary source operations. It is clear that if $\mathbf{P}$ reserves a fraction of its bandwidth for $\mathbf{S}$ 's transmission, the latter source would benefit. However, $\mathbf{P}$ is thereby reducing its access rate, and potentially its throughput. On the other hand, in exchange, $\mathbf{P}$ gets increased opportunities for cooperation by $\mathbf{S}$. It is the trade-off between these effects which we will examine in the numerical results..

Our protocol effectively selects the slots to be left unused by the primary source based only on locally available information, without requiring any coordination message with the secondary node (unlike [10]). The aim is to minimize the performance loss at the primary source due to bandwidth reduction, and to get the most from secondary source cooperation. Let us observe the following. A successful cooperative transmission, in which $\mathbf{S}$ delivers a packet from $\mathbf{P}$ to $\mathbf{D}$, avoids a potentially long sequence of retransmissions of this packet. Assuming $M$ is large enough to consider the channel coefficients between two consecutive retransmissions as independent, the number of retransmissions follows a geometric distribution according to the average failure probability. Thus, a single successful cooperative transmission saves the primary a whole sequence of primary retransmissions. 
Our protocol prescribes that, upon the reception of an ACK associated with a cooperative transmission, $\mathbf{P}$ leaves unused the slot scheduled for the retransmission of the packet delivered by $\mathbf{S}$. This way, $\mathbf{P}$ releases channel resource only upon recognizing a very likely gain from cooperation (since that was the case in the previous slot and fading is correlated). Moreover, $\mathbf{S}$ gains the opportunity to access the channel and transmits its own packets.

However, this is clearly not sufficient. In fact, this mechanism would also have diminishing returns as the primary source channel occupation increases (since the opportunities for secondary transmission, and thus cooperation, decrease). We exploit channel correlation in order to foster the virtuous resource allocation afforded by the mechanism described above. In particular, we define an SNR threshold $\tau^{*}<\tau\left(R_{p}\right)$. The primary source measures the perceived SNR at each packet sent by $\mathbf{D}$, and if this is below $\tau^{*}$ and a retransmission was not already scheduled then it forces itself to be idle in the next slot. ${ }^{1}$ Simply speaking, $\mathbf{P}$ recognizes that, if the measured channel conditions are below $\tau^{*}$, due to channel correlation, it is unlikely that the channel will be good enough to have a packet successfully delivery in the next slot.

To sum up, the primary source leaves unused slots in such a way that: a) it increases the chance of performance gains from cooperation; b) it limits the potential performance loss in giving up a transmission slot.

\section{MARKov Model OF THE Network}

Due to lack of space, we describe in detail the Markov chain associated with the coordinated scenario, as the noncooperative and the cooperative scenarios can be obtained by appropriately simplifying this case. We have first to derive for each of the links of the topology the associated channel chain, that is, the Markov chain describing the state and the evolution of the channel state. Starting from the channel chains, we derive the system chain, i.e., the Markov chain describing the status of the system.

For the sake of simplicity, we make some assumptions regarding the traffic dynamics at the nodes, that can be in principle removed at the cost of an increase in the model complexity. In particular, we assume the primary source transmits in an idle slot with fixed probability $\nu$ and the secondary source in saturation condition, i.e., $\mathbf{S}$ always has a packet to transmit. Note that in this setting, each slot is occupied by either $\mathbf{P}$ or $\mathbf{S}$. We also assume the secondary source has $Q$ possible transmission rates, defined as $\left\{R_{s}(1), R_{s}(2) \ldots, R_{s}(Q)\right\}=\left\{R_{p}, 2 R_{p}, \ldots, Q R_{p}\right\}$.

\section{A. Channel Chains}

We have to characterize the links between $\mathbf{S}$ and $\mathbf{D}, \mathbf{S}$ and $\mathbf{C}$ and $\mathbf{C}$ and $\mathbf{D}$. To this aim, we define three Markov chains,

\footnotetext{
${ }^{1}$ We can further simplify this mechanism, by asking $\mathbf{P}$ to leave the next slot idle upon the reception of a NACK.
}

denoted with $\Psi_{\mathrm{pd}}, \Psi_{\mathrm{ps}}$ and $\Psi_{\mathrm{sd}}$ where the subscripts denote the pair of nodes. The channel chain $\Psi_{x_{1} x_{2}}$ describes the state of the link through an integer index in $\left\{1, \ldots, N_{x_{1} x_{2}}\right\}$, where $N_{x_{1} x_{2}}$ is the number of the states of the chain. State $z$ corresponds to the region of $\operatorname{SNR}\left\{\tau_{z}, \tau_{z+1}\right\}$. Thus, to each chain there is an associated vector of $N_{x_{1} x_{2}}+1$ SNR thresholds defined as $\mathbf{v}_{x_{1} x_{2}}=\left\{0, \tau_{x_{1} x_{2}}(1), \ldots, \tau_{x_{1} x_{2}}\left(N_{x_{1} x_{2}}-1\right),+\infty\right\}$.

In order to describe the state between $\mathbf{P}$ and $\mathbf{D}$, we need $N_{\mathrm{pd}}=3$ states, with thresholds $\mathbf{v}_{p d}=\left\{0, \tau^{*}, \tau\left(R_{p}\right),+\infty\right\}$. Thus state 1,2 and 3 are associated with channel conditions below threshold $\tau^{*}$ and packet failure; channel conditions above threshold $\tau^{*}$ and packet failure; and channel conditions above threshold $\tau^{*}$ and packet success.

The link between $\mathbf{P}$ and $\mathbf{S}$ needs only to track packet decoding at the secondary source, and thus needs two states with thresholds $\mathbf{v}_{p s}=\left\{0, \tau\left(R_{p}\right),+\infty\right\}$ (recall that $R_{p}$ is fixed). Finally, the link between $\mathbf{S}$ and $\mathbf{D}$ is described by $Q+1$ states with thresholds $\mathbf{v}_{s d}=\left\{0, \tau\left(R_{s}(1)\right), \ldots, \tau\left(R_{s}(Q)\right),+\infty\right\}$. Assuming a capacity achieving model for packet decoding, the decoding thresholds are given by $\tau(R)=2^{R}-1$. We assume exponential path-loss with exponent $\alpha$, and fading coefficients are modeled as correlated Rayleigh fading variables with correlation $\rho$. Once fixed the distances between the nodes of the system as $d_{\mathrm{pd}}, d_{\mathrm{sd}}$ and $d_{\mathrm{ps}}$, we define the average SNRs $S_{x_{1} x_{2}}=\left(P d_{x_{1} x_{2}}^{-\alpha}\right) / N_{0}$, where $P$ and $N_{0}$ are the transmitted and the noise power, respectively.

As a result of the assumptions above, we can derive the transition probability between states $i$ and $j$ for the chain $\Psi_{x_{1} x_{2}}$ as in (1) where $\mathcal{I}_{0}(\cdot)$ is the modified Bessel function of the first kind and order zero, and the integration limits $b_{x_{1} x_{2}}(k)$ and the stationary distribution $\Theta_{x_{1} x_{2}}(i)$ are defined by

$$
\begin{aligned}
& b_{x_{1} x_{2}}(k)=\sqrt{\mathbf{v}_{x_{1} x_{2}}(k+1) / S_{x_{1} x_{2}}} \\
& \Theta_{x_{1} x_{2}}(i)=e^{\mathbf{v}_{x_{1} x_{2}}(i+1) / S}-e^{\mathbf{v}_{x_{1} x_{2}}(i) / S} .
\end{aligned}
$$

\section{B. System Chain}

In can be seen that, in order to fully account for the system dynamics (i.e., packet retransmissions) via a Markov model, we need to track a window of $M$ slots, that defines the overall state of the system [11], plus some additional variables. Then at each transition, corresponding to a temporal advancement of one slot, we shift left the window and the oldest slot exits the state, while a new slot enters the window (see Fig. 2).

We define the state of the system at slot $T$ as $\phi(T)=\left\{\mathbf{u}(T), \psi_{\mathrm{pd}}(T), \psi_{\mathrm{ps}}(T), \psi_{\mathrm{sd}}(T), \ell(T)\right\}$, where $\psi_{\mathrm{pd}}$, $\psi_{\mathrm{ps}}, \psi_{\mathrm{sd}}$ are the states of the channels in slot $T$ and $\ell(T)$ is the position in the window of the last packet failed by the primary source and decoded by the secondary source, i.e., the packet in the cooperation-buffer of $\mathbf{S}$. We set $\ell(T)=0$ if the cooperation-buffer is empty. $\mathbf{u}(T)=\left\{u_{1}, \ldots, u_{M}\right\}$ is the vector tracking the state of the slots in the window.

We now need to identify the set of the possible states of each slot. We adopt the following encoding:

- $u_{w}=0$, successful transmission by $\mathbf{P}$,

$$
\mathcal{Z}_{x_{1}, x_{2}}(i, j)=\int_{b_{x_{1} x_{2}}(i)}^{b_{x_{1} x_{2}}(i+1)} \int_{b_{x_{1} x_{2}}(j)}^{b_{x_{1} x_{2}}(j+1)} \frac{4 c_{1} c_{2}}{1-\rho} e^{-\frac{c_{1}^{2}+c_{2}^{2}}{1-\rho}} \mathcal{I}_{0}\left(\frac{2 \sqrt{\rho} c_{1} c_{2}}{1-\rho}\right) \mathrm{d} c_{1} \mathrm{~d} c_{2} / \Theta_{x_{1} x_{2}}(i)
$$




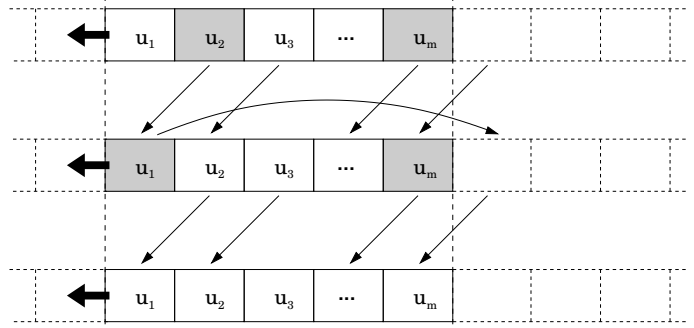

Fig. 2. Sliding window Markov model of the primary source in isolation. When we consider slot $T, u_{t}$ represents the state of slot $T-M+t$.

- $u_{w}=1$, failed transmission by $\mathbf{P}$, but packet delivered through cooperation,

- $u_{w}=2$, failed transmission by $\mathbf{P}$ and packet not delivered through cooperation,

- $u_{w}=3, \ldots, Q+3$, the secondary source delivered data from its own queue at rate $R_{s}\left(u_{w}-2\right)$,

- $u_{W}=Q+4$, failed transmission from the secondary source.

This representation is sufficient to evolve the chain and collect the throughput in [bits/s/Hz] achieved by the primary and the secondary source.

As stated before, during a transition the vector $\mathbf{u}(T)$ is shifted and we thus set $u_{w}(T+1)=u_{w+1}(T), w=1, \ldots, M-1$. The distribution state of the slot entering the window, that is, $u_{M}(T+1)$ is entirely defined by $u_{1}(T)$ and the state of the various channels and $\ell$. Due to cooperation, if $\ell(T)>0$ during a transition also the value of $u_{\ell-1}(T+1)$ may be different from $u_{\ell}(T)$.

Let us define transition probabilities focusing on the part of the state not trivially shifted. We then define the vector $\mathbf{n}(T+1)=\left\{u_{M}(T+1), u_{\ell-1}(T+1), \psi_{\mathrm{pd}}(T+1), \psi_{\mathrm{ps}}(T+\right.$ $\left.1), \psi_{\text {sd }}(T+1), \ell(T+1)\right\}$ and we look at its value in the next slot. ${ }^{2}$ If $u_{1}(T)=3$ the primary source will retransmit the packet in the entering slot, and we report in (4) the values and probabilities for the previously introduced vector in this case. Thus, if the channel between $\mathbf{P}$ and $\mathbf{D}$ is equal to 2 , then the transmission is successful and $\ell$ keeps its value. If $\psi_{\mathrm{pd}}<2$, i.e., the transmission fails, we need to distinguish between two cases: if $\psi_{\mathrm{ps}}=1$, then $\mathbf{S}$ stores the packet in its buffer, and then $\ell(T+1)=M$, otherwise $\ell(T+1)=\ell T$.

If $u_{1}(T) \neq 3$ and $\psi_{p d}=0$, then $\mathbf{P}$ leaves the associated slot idle, and the secondary source transmits. If $\ell(T)=0$ (see

\footnotetext{
${ }^{2}$ With a slight abuse of notation we wil say that $u_{\ell-1}=u_{\ell}(T)$ even when $\ell=0$.
}

Eq. (5)), then $\mathbf{S}$ transmits only packets from its own queue. $\mathbf{S}$ achieves a success, and its rate is $\max \left(R_{s}\left(\psi_{\mathrm{sd}}(T)-1\right), R_{s}(1)\right)$ if the new state of $\Psi_{\mathrm{sd}}$ in slot $T+1$ is greater than or equal to that of slot $T$. Otherwise transmission by source $\mathbf{S}$ fails. If $\ell>0$ (see Eq. (6)), then $\mathbf{S}$ cooperates. Packet failure/success has the same probability of the case with $\ell=0$, but if the packet succeeds, then the rate achieved by $\mathbf{S}$ is reduced due to transmission of the packet in the cooperation-buffer and $u_{\ell(T)}$ is set to 1 .

The values and probabilities associated to the vector $\mathbf{n}(T+1)$ for this case are reported in (5). The same holds for $u_{1}(T)=2$, where the primary source keeps idle to remunerate $\mathbf{S}$ of the successful delivery.

In the other cases, the primary transmits with probability $\nu$. Thus, $\mathbf{n}(T+1)$ takes the values in Eq. (4) with the associated probabilities multiplied by $\nu$ and the values in (5) with the associated probabilities multiplied by $1-\nu$

\section{NumERICAL RESUlTS}

In this Section, we show numerical results obtained through the previously presented analytical framework. We compare the non-cooperative, the cooperative and the coordinated case in terms of throughput achieved by the primary and the secondary source, denoted with $\mathcal{T}_{p}$ and $\mathcal{T}_{s} . \mathcal{T}_{p}$ and $\mathcal{T}_{s}$ can be obtained from the stationary distribution of the chain $\pi(\phi)$ as $\mathcal{T}_{p}=\sum_{\mathcal{X}_{p}} \pi(\phi) R_{p}$ and $\mathcal{T}_{p}=\sum_{\mathcal{X}_{s}} \pi(\phi) R_{s}\left(u_{1}\right)$, respectively, where $\mathcal{X}_{p}$ and $\mathcal{X}_{p}$ are the sets of states of the system in which $u_{1}=0$ or $u_{1}=1$ and $u_{1}=3, \ldots, Q+3$.

We consider an aligned topology where the distance between $\mathbf{P}$ and $\mathbf{S}$ is $d_{\mathrm{ps}}=100 \mathrm{~m}$, and $d_{\mathrm{cd}}=d_{\mathrm{pd}}-d_{\mathrm{sd}}$. The transmitted and noise power are set to $P=-6 \mathrm{~dB}$ and $N_{0}=-$ $60 \mathrm{~dB}$, respectively. The attenuation exponent is $\alpha=3.5$. The primary source transmits at rate $R_{p}=1 \mathrm{bit} / \mathrm{s} / \mathrm{Hz}$, and we set $Q=4$. Thus, the secondary source has a set of rates equal to $\left\{R_{p}, 2 R_{p}, 3 R_{p}, 4 R_{p}\right\} . \tau^{*}$ is set such that the stationary probability of state 0 for the channel between $\mathbf{P}$ and $\mathbf{D}$ is equal to 0.1 .

Fig. 3 depicts the throughput of the primary and secondary sources for the three considered scenarios as a function of the distance between $\mathbf{P}$ and $\mathbf{S}$ for $\nu=0.6$. It can be easily understood that the closer $\mathbf{P}$ to $\mathbf{S}$ the higher the probability that the latter decodes packets that were sent by the former and received a NACK. Nevertheless, the closer $\mathbf{S}$ to $\mathbf{D}$, the higher the probability the former successfully relays a packet from $\mathbf{P}$. Thus, the gain provided by cooperation increases when $\mathbf{S}$

$$
\begin{array}{r} 
\begin{cases}\left\{0, u_{\ell(T)}(T), 2, \psi_{\mathrm{ps}}(T+1), \psi_{\mathrm{sd}}(T+1), \ell\right\} & \mathcal{Z}_{p d}\left(\psi_{\mathrm{pd}}(T), 2\right) \mathcal{Z}_{p s}\left(\psi_{\mathrm{ps}}(T), \psi_{\mathrm{ps}}(T+1)\right) \mathcal{Z}_{s d}\left(\psi_{\mathrm{sd}}(T), \psi_{\mathrm{sd}}(T+1)\right) \\
\left\{2, u_{\ell(T)}(T), 0,1, \psi_{\mathrm{sd}}(T+1), M\right\} & \mathcal{Z}_{p d}\left(\psi_{\mathrm{pd}}(T), 0\right) \mathcal{Z}_{p s}\left(\psi_{\mathrm{ps}}(T), 1\right) \mathcal{Z}_{s d}\left(\psi_{\mathrm{sd}}(T), \psi_{\mathrm{sd}}(T+1)\right) \\
\left\{2, u_{\ell(T)}(T), 0,0, \psi_{\mathrm{sd}}(T+1), \ell\right\} & \mathcal{Z}_{p d}\left(\psi_{\mathrm{pd}}(T), 0\right) \mathcal{Z}_{p s}\left(\psi_{\mathrm{ps}}(T), 0\right) \mathcal{Z}_{s d}\left(\psi_{\mathrm{sd}}(T), \psi_{\mathrm{sd}}(T+1)\right) \\
\left\{2, u_{\ell(T)}(T), 1,1, \psi_{\mathrm{sd}}(T+1), M\right\} & \mathcal{Z}_{p d}\left(\psi_{\mathrm{pd}}(T), 2\right) \mathcal{Z}_{p s}\left(\psi_{\mathrm{ps}}(T), 1\right) \mathcal{Z}_{s d}\left(\psi_{\mathrm{sd}}(T), \psi_{\mathrm{sd}}(T+1)\right) \\
\left\{2, u_{\ell(T)}(T), 1,0, \psi_{\mathrm{sd}}(T+1), \ell\right\} & \mathcal{Z}_{p d}\left(\psi_{\mathrm{pd}}(T), 2\right) \mathcal{Z}_{p s}\left(\psi_{\mathrm{ps}}(T), 0\right) \mathcal{Z}_{s d}\left(\psi_{\mathrm{sd}}(T), \psi_{\mathrm{sd}}(T+1)\right)\end{cases} \\
\ell=0 \begin{cases}\left\{Q+4, u_{\ell(T)}(T), \psi_{\mathrm{pd}}(T+1), \psi_{\mathrm{ps}}(T+1)<\max \left(2, \psi_{\mathrm{ps}}(T+1)-1\right), \psi_{\mathrm{sd}}(T+1), \ell\right\} & \prod \mathcal{Z}_{x_{1}, x_{2}}\left(\psi_{\mathrm{x}_{1} \mathrm{x}_{2}}(T), \psi_{\mathrm{x}_{1} \mathrm{x}_{2}}(T+1)\right) \\
\left\{\psi_{\mathrm{sd}}(T), u_{\ell(T)}(T), \psi_{\mathrm{pd}}(T+1), \psi_{\mathrm{ps}}(T+1) \geq \max \left(2, \psi_{\mathrm{ps}}(T+1)-1\right), \psi_{\mathrm{sd}}(T+1), \ell\right\} & \prod \mathcal{Z}_{x_{1}, x_{2}}\left(\psi_{\mathrm{x}_{1} \mathrm{x}_{2}}(T), \psi_{\mathrm{x}_{1} \mathrm{x}_{2}}(T+1)\right)\end{cases} \\
\ell>0 \begin{cases}\left\{Q+4, u_{\ell(T)}(T), \psi_{\mathrm{pd}}(T+1), \psi_{\mathrm{ps}}(T+1)<\max \left(2, \psi_{\mathrm{ps}}(T+1)-1\right), \psi_{\mathrm{sd}}(T+1), \ell\right\} & \prod \mathcal{Z}_{x_{1}, x_{2}}\left(\psi_{\mathrm{x}_{1} \mathrm{x}_{2}}(T), \psi_{\mathrm{x}_{1} \mathrm{x}_{2}}(T+1)\right) \\
\left\{\psi_{\mathrm{sd}}(T)-1,1, \psi_{\mathrm{pd}}(T+1), \psi_{\mathrm{ps}}(T+1) \geq \max \left(2, \psi_{\mathrm{ps}}(T+1)-1\right), \psi_{\mathrm{sd}}(T+1), 0\right\} & \prod \mathcal{Z}_{x_{1}, x_{2}}\left(\psi_{\mathrm{x}_{1} \mathrm{x}_{2}}(T), \psi_{\mathrm{x}_{1} \mathrm{x}_{2}}(T+1)\right)\end{cases}
\end{array}
$$




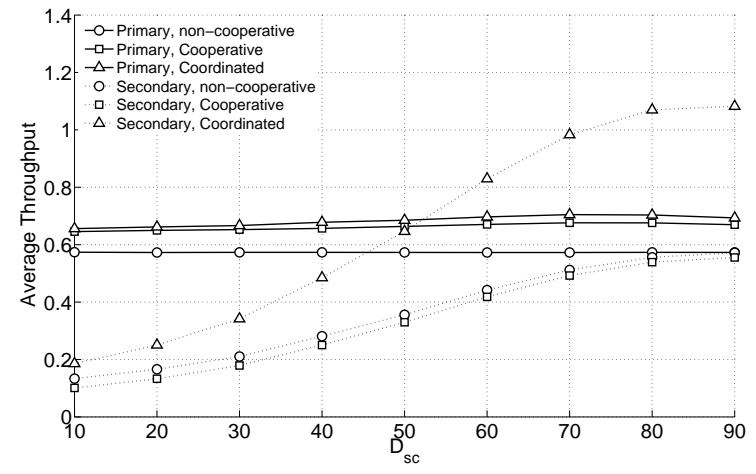

Fig. 3. Average throughput achieved by the various protocols as a function of the distance between the primary and the secondary source. The transmission probability of $\mathbf{P}$ is $\nu=0.6$

moves away from $\mathbf{P}$, but slightly decreases when the former gets too close to the destination.

The gain provided by the cooperative scenario to the primary source is between $10 \%$ and $20 \%$. It can be observed that cooperation causes a small decrease of the throughput of $\mathbf{S}$ that is sacrificing part of its available rate to cooperate with $\mathbf{P}$. If we introduce coordination, the gain due to cooperation is only slightly decreased, and the region of $d_{\mathrm{ps}}$ with the highest reduction occurs where cooperation is less profitable. On the other hand, the throughput achieved by the secondary source is boosted by coordination, resulting in a significant incentive to cooperate. The throughput gain at the secondary source due to coordination is particularly evident when $\mathbf{S}$ is close to $\mathbf{D}$, i.e., where rate adaptation allows the secondary source to transmit at high rate more often.

In Fig. 4, the same comparison is shown for different values of the transmission probability of the primary source $\nu$ when $d_{\mathrm{ps}}=60 \mathrm{~m}$. The gain with respect to the non cooperative case is shown in Fig. 5. The throughput gain in the coordinated case is smaller than that achieved with a pure cooperative case, especially when the primary access rate is small. Nevertheless, in this case coordination is not necessary, as the secondary source achieves a satisfactory throughput and thus is encouraged to cooperate even in exchange of access only. As $\nu$ is increased, the gains provided by simple cooperation and coordination get closer (and the latter overcomes the former around $\nu=0.85$ ), as the secondary source cannot effectively cooperate in the former case due to primary transmissions. On the other hand, in the non-cooperative and cooperative cases the secondary source sees its throughput decrease as $\nu$ is increased, while in the coordinated case it can access the channel in a significant fraction of the slots.

\section{Conclusions}

We presented an analytical framework based on Markov chain theory for the analysis of cooperative cognitive networks with packet retransmission at primary sources and time correlated fading. We showed that a simple collaboration behavior implemented at the primary sources that exploits the fading memory can preserve the gain provided by secondary sources' cooperation, while boosting the performance of the latter class of users.

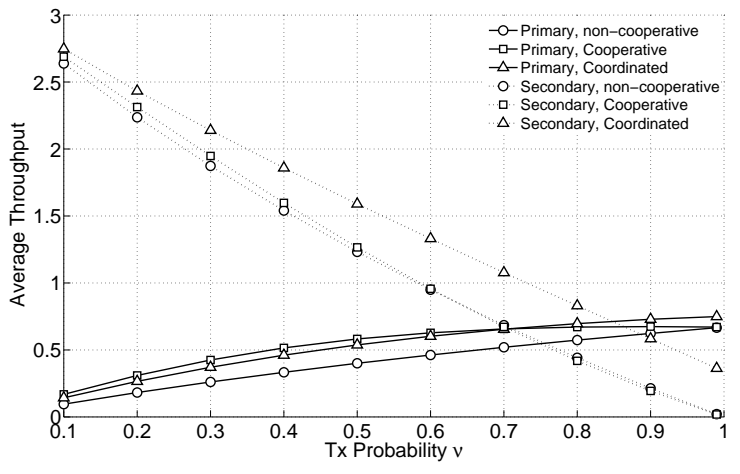

Fig. 4. Average throughput achieved by the various protocols as a function of the transmission probability of the primary source. $d_{\mathrm{ps}}$ is set to $60 \mathrm{~m}$.

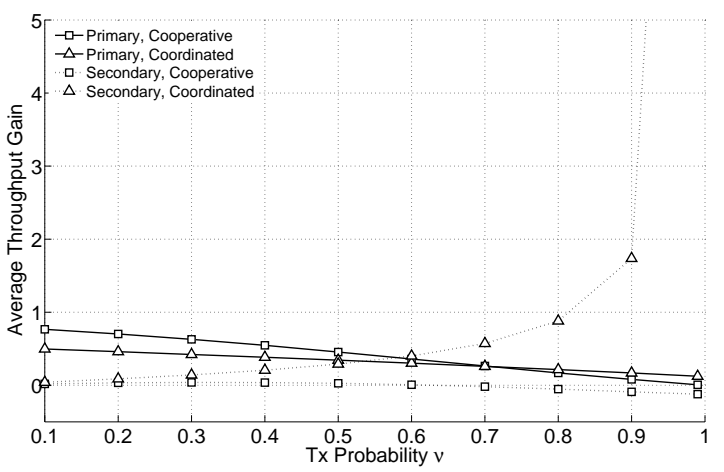

Fig. 5. Average throughput gain with respect to the non-cooperative case for the cooperative and the coordinated protocols as a function of the transmission probability of the primary source. $d_{\mathrm{ps}}$ is set to $60 \mathrm{~m}$.

\section{REFERENCES}

[1] J. Mitola, "Cognitive radio: an integrated agent architecture for softwaredefined radio," Doctor of Technology, Royal Inst. Technol. (KTH), Stockholm, Sweden, 2000.

[2] Q. Zhao and L. Tong and A. Swami and Y. Chen, "Decentralized cognitive MAC for opportunistic spectrum access in ad hoc networks: a POMPD framework," IEEE J. Select. Areas Commun., vol. 25, no. 3, pp. 589-600, Apr. 2007.

[3] S. Geirhofer and L. Tong and B. M. Sadler, "Dynamic Spectrum access in the time domain: modeling and exploiting white space," IEEE Commun. Mag., vol. 45, no. 5, pp. 66-87, May 2007.

[4] R. Urgaonkar and M. J. Neely, "Opportunistic scheduling with reliability guarantees in cognitive radio networks," in Proc. of the 27th IEEE Conf. on Computer Communications (IEEE INFOCOM), Phoenix, AZ, USA, Apr. 2008, pp. 1301-1309.

[5] M. Levorato, U. Mitra, and M. Zorzi, "On optimal control of wireless networks with multiuser detection, hybrid ARQ and distortion constraints," in Proc. of the 28th IEEE Conf. on Computer Communications (IEEE INFOCOM), Rio de Janeiro, Brazil, Apr. 2009.

[6] M. Levorato, O. Simeone, and U. Mitra, "Interference management via rate splitting and HARQ over time-varying fading channels," in Proc. of ACM Coronet (ACM Mobicom symposium), 2009, Beijing, Cina, 2009.

[7] O. Simeone, Y. Bar-Ness, and U. Spagnolini, Cooperative cognitive communications. Cooperative wireless communications, Auerbach Publications, CRC Press, 2009.

[8] A. Sendonaris, E. Erkip, and B. Aazhang, "User cooperation diversity, part I: system description," IEEE Trans. Commun., vol. 51, pp. 19271938, Nov. 2003.

[9] O. Simeone, I. Stanojev, S. Savazzi, Y. Bar-Ness, and R. Pickholz, "Spectrum leasing to cooperating secondary ad hoc networks," IEEE J. Select. Areas Commun., vol. 26, no. 1, pp. 203-213, Jan. 2008.

[10] I. Stanojev, O. Simeone, U. Spagnolini, R. Pickholtz, and Y. Bar-Ness, "An auction-based incentive mechanism for non-altruistic cooperative arq via spectrum-leasing," in Proc. of IEEE Global Commun. Conf. (GLOBECOM), Honolulu, Hawaii, USA, Nov. 30-Dec. 42009.

[11] M. Rossi, L. Badia, and M. Zorzi, "On the delay statistics of SR ARQ over Markov channels with finite round-trip delay," IEEE Trans. Wireless Commun., vol. 4, no. 4, pp. 1858-1868, July 2005. 